\title{
THE CAYLEY GRAPHS OF COXETER AND ARTIN GROUPS
}

\author{
CARL DROMS AND HERMAN SERVATIUS
}

(Communicated by Ron Solomon)

\begin{abstract}
We obtain a complete classification of the Coxeter and Artin groups whose Cayley graphs with respect to the standard presentations are planar. We also classify those whose Cayley graphs have planar embeddings in which the vertices have no accumulation points.
\end{abstract}

\section{INTRODUCTION}

Let $A \leftrightarrow^{q} B$ denote the relation $A B A B A \cdots=B A B A B \cdots$ with $q$ letters on each side of the equals sign, so that, in particular, $A \leftrightarrow^{1} B$ means $A=B$, $A \leftrightarrow^{2 r+1} B$ means $(A B)^{r} A=(B A)^{r} B$, and $A \leftrightarrow^{2 r} B$ means $(A B)^{r}=(B A)^{r}$. We also set $A \leftrightarrow^{\infty} B$ to be the empty relation. In this notation (see [2]), Artin's braid group on $n+1$ strings, $B(n+1)$, with generators $\sigma_{1}, \sigma_{2}, \cdots \sigma_{n}$, has the defining relations

$$
\sigma_{\mu} \stackrel{3}{\leftrightarrow} \sigma_{\mu+1} \quad \text { and } \quad \sigma_{\mu} \stackrel{2}{\leftrightarrow} \sigma_{\nu} \quad(\nu>\mu+1) .
$$

More generally, given a symmetric $n \times n$ integer matrix $M=\left(m_{i j}\right)$ with $2 \leq$ $m_{i j} \leq \infty$ for $i \neq j$ and $m_{i i}=1$, the Artin group $\mathscr{A}(M)$ is the group with $n$ generators $\left\{s_{1}, \ldots, s_{n}\right\}$ and defining relations $s_{i} \leftrightarrow^{m_{i j}} s_{j}$ for all $i \neq j$. Practically, $M$ may be specified by labeling the edges of the complete graph on $n$ vertices with values in $\{2, \ldots, \infty\}$. Many Artin groups, including the braid groups, are realized as the fundamental groups of hyperplane complements in a complex vector space $[1,3]$. An important homomorphic image of $\mathscr{A}(M)$ is $\mathscr{C}(M)$, the Coxeter group on $M$, which is obtained from $\mathscr{A}(M)$ by adding the $n$ relations $s_{i}^{2}=1$. Coxeter groups are realized as subgroups of $\mathrm{GL}(m, R)$, and for a large class, including all those which are finite, the generators can be taken to be reflections in a set of hyperplanes in $R^{m}$. Many Coxeter groups act on $S^{2}, R^{2}$, or $H^{2}$-the sphere, the euclidean plane, and hyperbolic plane respectively - as groups of isometries. Choosing a fundamental region $F$ for the action exhibits $\mathscr{C}(M)$ as a group of symmetries of the tiling $\{g F \mid g \in$ $\mathscr{C}(M)\}$. For example,

Received by the editors October 24, 1991; the contents of this paper were presented at the 1991 SUNY-Albany Conference on Topology and Group Theory, October 12, 1991.

1991 Mathematics Subject Classification. Primary 20F55; Secondary 20F36, 05 C25.

Key words and phrases. Coxeter group, Artin group, planar graph. 
Proposition 1 [4, p. 58]. If $m_{i, j}=\infty$ for $|j-i| \geq 2$ (indices $\bmod n$ ), then $\mathscr{C}(M)$ is the group generated by reflections in the sides of an $n$-gon with angles $\pi / m_{i, i+1}, i=1, \ldots, n(\bmod n)$, which lies in $S^{2}, R^{2}$, or $H^{2}$, depending on the sizes of the angles $\pi / m_{i, i+1}$.

In this setting, the tiles sharing an edge with $F$ are $\left\{s_{i} F\right\}$, so the tiles sharing an edge with $g F$ are $\left\{g s_{i} F\right\}$, and placing a vertex labeled $g$ in the center of each $g F$ and an edge labeled $s_{i}$ connected $g$ and $g s_{i}$ constructs the Cayley graph of $\mathscr{C}(M)$ with respect to $\left\{s_{i}\right\}$ as the planar dual to the tiling $\{g F\}$. Note that the Cayley graph is drawn with a single unoriented edge joining $g$ and $g s_{i}$ as is customary when the generators are involutions.

It is natural to ask whether a Coxeter group whose Cayley graph with respect to the standard generating set is planar acts as a group of isometries of the dual graph, properly embedded. As we shall see, this is not the case.

In this article we will characterize those Coxeter groups whose Cayley graphs are planar with respect to the standard generating set, as well as those whose Cayley graphs can be embedded in the plane in such a way that there are no accumulation points of vertices.

\section{COXETER GROUPS}

A Coxeter matrix $M$ is traditionally indicated by labeling the edges of the complete graph on the $n$ vertices $\left\{s_{1}, \ldots, s_{n}\right\}$ such that the edge $\left(s_{i}, s_{j}\right)$ has weight $m_{i, j}$, consistent with the notation $s_{i} \leftrightarrow^{m_{i, j}} s_{j}$.

Let $M$ be a complete graph each of whose edges is labeled with an integer $\geq 2$ or $\infty$. When, as is classically done, the edges of weight 2 are erased, the graph $M_{2}$ is called the Coxeter graph. The justification for this is that the Coxeter group is the direct product of the Coxeter groups on the connected components of its Coxeter graph.

In this paper, however, it will be convenient to erase from $M$ only those edges with weight $\infty$, forming a graph denoted by $M_{\infty}$. We prove the following result.

Theorem 1. $\mathscr{C}(M)$ has a planar Cayley graph with respect to the standard generators if and only if the graph $M_{\infty}$ is outer planar.

A graph $\Gamma$ is called outer planar if it can be drawn in the plane in such a way that all vertices lie on the boundary of a single face. We call an edge of $\Gamma$ a boundary edge (relative to the given planar embedding) if it forms part of the boundary of this face. The other edges are called interior edges.

Note that $\Gamma$ is outer planar if and only if the graph $\Gamma^{*}$ obtained from $\Gamma$ by adjoining a new vertex that is adjacent to every vertex of $\Gamma$ is planar.

Proof. Let $G$ be the Cayley graph of $\mathscr{C}(M)$, and suppose that $G$ is planar. Let $s_{1}, \ldots, s_{n}$ be the vertices of $M$. For each pair $s_{i}, s_{j}$ of vertices that are adjacent in $M_{\infty}$, the $\left(s_{i}, s_{j}\right)$-polygon in $G$ will consist of the vertices $\left\{1, s_{i}, s_{i} s_{j}, s_{i} s_{j} s_{i}, \ldots,\left(s_{i} s_{j}\right)^{m_{i j}}=1\right\}$, together with the edges joining them. Any two such polygons intersect only along the edge $\left(1, s_{i}\right)$ or $\left(1, s_{j}\right)$. Consider the subgraph $G_{1}$ of $G$ consisting of the $\left(s_{i}, s_{j}\right)$-polygons, together with all vertices $s_{i}$ corresponding to isolated vertices of $M_{\infty}$. If we replace each of the paths $\left\{s_{i}, s_{i} s_{j}, \ldots, s_{j}\right\}$ by a single edge, the resulting graph is homeomorphic to $G_{1}$ and isomorphic to $M_{\infty}^{*}$, with 1 being the additional vertex. Thus, since $G_{1}$ is planar, $M_{\infty}$ is outer planar. 
For the converse, we first embed $M_{\infty}$ in a maximal outer planar graph $\Gamma_{M}$ by adding edges labeled $\infty$ to $M_{\infty}$, if necessary. $\Gamma_{M}$ is a triangulation of a polygon [5].

We will show that the Cayley graph of $\mathscr{C}(M)$ has a planar embedding with the following property:

If the vertices $s_{i}$ and $s_{j}$ are joined in $\Gamma_{M}$ by a boundary edge with a finite label, then every $\left(s_{i}, s_{j}\right)$-polygon is the boundary of a face.

Note that if the graphs $\Gamma$ and $\Sigma$ are planar, and if $\gamma$ and $\sigma$ are $n$-gons in $\Gamma$ and $\Sigma$, respectively, then the union of $\Gamma$ and $\Sigma$, with $\gamma$ and $\sigma$ identified, is planar. Thus, two planar graphs can be glued together along the boundaries of faces of equal perimeter to form a new planar graph.

If every interior edge of $\Gamma_{M}$ is labeled " $\infty$," then $M$ is an induced subgraph of a polygon, so by Proposition 1, the Cayley graph of $\mathscr{C}(M)$ has a planar embedding that is the planar dual of a geometric tiling, and this embedding has the property that if $s_{i}$ and $s_{j}$ are joined in $M$ by an edge with a finite label, then the $\left(s_{i}, s_{j}\right)$-polygons bound faces.

So suppose that some interior edge $s_{i}-s_{j}$ of $\Gamma_{M}$ has a finite label $n$. Then we can write $\Gamma_{M}$ as the union of two outer planar subgraphs $\Gamma_{1}$ and $\Gamma_{2}$ along this edge. Note that

1. Each of $\Gamma_{1}$ and $\Gamma_{2}$ is outer planar, has strictly fewer vertices than $\Gamma_{M}$, and the edge $s_{i}-s_{j}$ is a boundary edge of each.

2. Every boundary edge of $\Gamma$ is a boundary edge of either $\Gamma_{1}$ or $\Gamma_{2}$.

3. $\mathscr{C}(M)$ is the free product of the groups $\mathscr{E}\left(\Gamma_{1}\right)$ and $\mathscr{C}\left(\Gamma_{2}\right)$, amalgamating the subgroups generated by $\left\{s_{i}, s_{j}\right\}$.

Let $G_{1}$ and $G_{2}$ be planar embeddings of the Cayley graphs of $\mathscr{C}\left(\Gamma_{1}\right)$ and $\mathscr{C}\left(\Gamma_{2}\right)$, respectively, in which all polygons corresponding to boundary edges of $\Gamma_{1}$ or $\Gamma_{2}$ bound faces. Note in particular that all $\left(s_{i}, s_{j}\right)$-polygons in $G_{1}$ and $G_{2}$ bound faces.

We will construct a planar embedding of the Cayley graph $G$ of $\mathscr{C}(M)$ as follows: let $G^{(0)}=G_{1}$. Given $G^{(k)}$ with $k$ even (odd), we glue in a copy of $G_{1}$ $\left(G_{2}\right)$ along each $\left(s_{i}, s_{j}\right)$-polygon of $G^{(k)}$ that is the boundary of a face. Call the resulting graph $G^{(k+1)}$. Note that each $G^{(k)}$ is planar and that any polygon of $G^{(k)}$ corresponding to a boundary edge of $\Gamma_{M}$ bounds a face.

By (3) the graph $G=\lim G^{(k)}$ is the Cayley graph of $\mathscr{C}(M)$, and since each $G^{(k)}$ is planar, so is $G$. Finally, it is clear that any polygon in $G$ corresponding to a boundary edge of $M_{\infty}$ bounds a face, since this is the case in each $G^{(k)}$.

If $M_{\infty}$ is a $k$-gon or an induced subgraph of a $k$-gon, then by the remarks following Proposition 1, the Cayley graph can be embedded in the plane in such a way that there are no accumulation points of vertices.

We have

Theorem 2. The Cayley graph of $\mathscr{C}(M)$ with respect to $s_{1}, \ldots, s_{n}$ has a planar embedding without accumulation points of vertices if and only if $M_{\infty}$ is an induced subgraph of a polygon.

Proof. We have seen that the second condition implies the first. 
For the converse, suppose that $M_{\infty}$ is not an induced subgraph of a polygon, so that $M_{\infty}$ contains either (i) a vertex of degree three or more or (ii) the disjoint union of a vertex and a polygon. Let $G$ be the Cayley graph of $\mathscr{C}(M)$.

Suppose first that $M_{\infty}$ has a vertex of degree three or more, say $v \in M_{\infty}$ is adjacent to $x, y$, and $z$. Then one pair of these, say $(x, y)$, is not an edge in $M_{\infty}$, since otherwise $\{v, x, y, z\}$ would generate a complete graph, contradicting the outer planarity of $M_{\infty}$. Consider the following infinite paths in $G$,

$$
\begin{aligned}
& X=1, x, x y, x y x, x y x y, \ldots, \\
& Y=1, y, y x, y x y, y x y x, \ldots, \\
& Z=1, z, z x, z x y, z x y x, z x y x y, \ldots
\end{aligned}
$$

Since there are no accumulation points of vertices, these three paths divide the plane into three regions. However, none of these regions can contain $v$ since there is a finite path of the form $v, v w, v w v, \ldots, w$ for each $w \in\{x, y, z\}$ and one of $x, y$, or $z$ lies in the exterior of each region.

Suppose, on the other hand, $M_{\infty}$ contains an induced subgraph that is the disjoint union of the $k$-gon $\left\{s_{1}, \ldots, s_{k}\right\}, k \geq 3$, and a vertex $x$. For each $i$, let $P_{i}$ be the infinite path starting at 1 labeled $s_{i} x s_{i} x s_{i} x \ldots$. Since there are no accumulation points of vertices, these paths divide the plane into $k$ components. The path $\left\{s_{1}, s_{1} s_{2}, s_{1} s_{2} s_{1}, \ldots,\left(s_{1} s_{2}\right)^{m_{12}}\right\}$ intersects the paths $P_{i}$ only at $s_{1}$ and $s_{2}$, and so lies in the region bounded by $P_{1}$ and $P_{2}$. Similarly, the elements $s_{i} s_{i+1}$ and $s_{i+1} s_{i}$ lie in the region bounded by $P_{i}$ and $P_{i+1}$ (indices $\bmod k)$. Consider, however, the path $\left\{\left(s_{1}\right) s_{2},\left(s_{1}\right) s_{2} s_{3},\left(s_{1}\right) s_{2} s_{3} s_{2}, \ldots,\left(s_{1}\right) s_{3} s_{2}\right.$, $\left.\left(s_{1}\right) s_{3}\right\}$. It intersects no $P_{i}$, so $s_{1} s_{2}$ and $s_{1} s_{3}$ lie in the same component. Similarly, proceeding around the $k$-gon, $s_{1} s_{4}, \ldots, s_{1} s_{k}$ all lie in this component as well-a contradiction.

\section{ARTIN GROUPS}

Let $\mathscr{A}(M)$ be an Artin group. Adding relations setting all the generators equal to each other results in a free abelian group, and it follows that two positive words - that is, two words on the generators with no negative exponents-can represent the same element of $\mathscr{A}(M)$ only if they have the same length.

Lemma 1. If some edge of $M$ is labeled with an integer $q \geq 3$, then the Cayley graph of $\mathscr{A}(M)$ is not planar.

Proof. Suppose the vertices $a$ and $b$ are joined by an edge labeled $q \geq 3$, so $a b a \cdots=b a b \cdots$ with $q$ factors on each side. Let $\Delta$ denote the common value of these two expressions. Note that if $q$ is odd then $a \Delta=\Delta b$ and $b \Delta=\Delta a$, and if $q$ is even then $\Delta$ commutes with both $a$ and $b$.

Consider the vertices and paths in the Cayley graph of $\mathscr{A}(M)$ depicted in Figure 1, where the paths are those indicated by the labels.

By examining the lengths of the elements occurring on these paths, together with their images in the Coxeter group, one sees that they are all distinct, with the possible exception of $a b^{2}$ and $b^{2} a$. Thus, the corresponding subgraph of the Cayley graph of $\mathscr{A}(M)$ is isomorphic either to that in Figure 1 or to that pictured in Figure 2, neither of which is planar. 


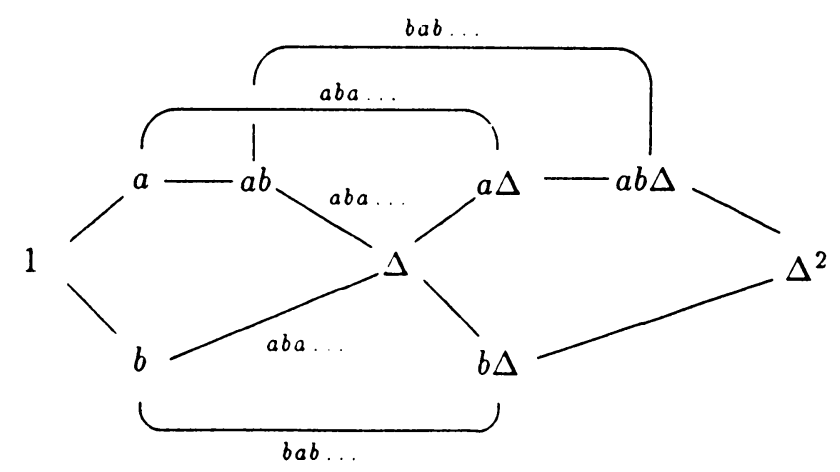

Figure 1

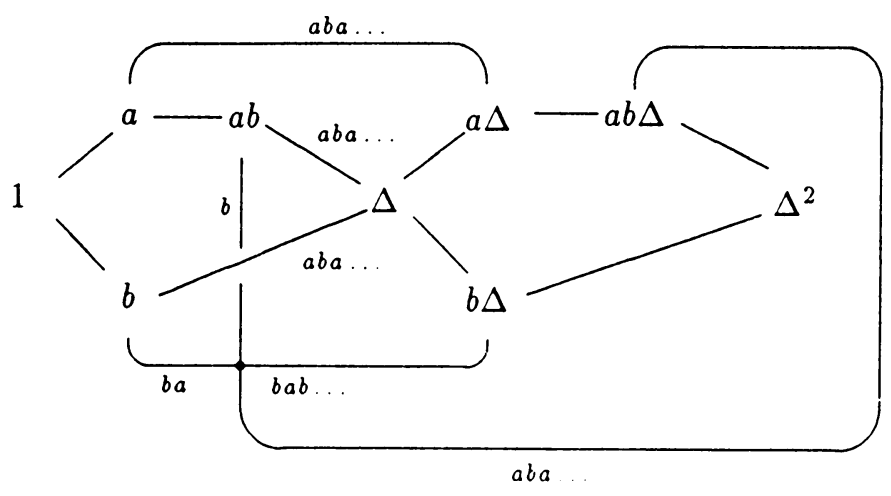

FIGURE 2

We now consider Artin groups defined by graphs whose edge labels are each either 2 or $\infty$.

Let $M$ be such a graph. Let $M^{2}$ be the double of $M$, that is, $M^{2}$ has two vertices $v^{(1)}$ and $v^{(2)}$ for each vertex $v$ of $M$, and if $v$ and $w$ are joined by an edge labeled $q$ in $M$, then each of $v^{(1)}, v^{(2)}$ is joined to each of $w^{(1)}, w^{(2)}$ by an edge labeled $q$ in $M^{2}$. Additionally, $v^{(1)}$ and $v^{(2)}$ are joined by an edge labeled $\infty$ for each vertex $v$ of $M$.

In the following lemma, we compare the Cayley graph of $\mathscr{A}(M)$ to that of $\mathscr{C}\left(M^{2}\right)$ (an undirected graph). When we do this, we are disregarding the fact that the former is a directed graph and looking only at its underlying graph structure.

Lemma 2. The Cayley graphs of $\mathscr{A}(M)$ and of $\mathscr{C}\left(M^{2}\right)$ are isomorphic as undirected graphs.

Proof. Given a word $W$ in the vertices of $M$ and a vertex $v$, we define the $v$-length of $W$, denoted $|W|_{v}$, to be the sum of all exponents of occurrences of powers of $v$ in $W$. 
We then define a function $\phi$ from the words in the vertices of $M$ to the words in the vertices of $M^{2}$ as follows:

$$
\begin{aligned}
\phi(1) & =1 ; \\
\phi(W v) & = \begin{cases}\phi(W) v^{(1)} & \text { if }|W|_{v} \text { is even, } \\
\phi(W) v^{(2)} & \text { if }|W|_{v} \text { is odd },\end{cases} \\
\phi\left(W v^{-1}\right) & = \begin{cases}\phi(W) v^{(2)} & \text { if }|W|_{v} \text { is even, } \\
\phi(W) v^{(1)} & \text { if }|W|_{v} \text { is odd } .\end{cases}
\end{aligned}
$$

Now a computation shows that $\phi$ actually defines a bijection from elements of $\mathscr{A}(M)$ to those of $\mathscr{C}\left(M^{2}\right)$; that is, if $W$ and $W^{\prime}$ represent the same element of $\mathscr{A}(M)$, then $\phi(W)$ and $\phi\left(W^{\prime}\right)$ represent the same element of $\mathscr{C}\left(M^{2}\right)$. Finally, since the Cayley graphs are drawn with respect to the elements $v$ (resp. $\left.v^{(1)}, v^{(2)}\right)$, we see that $\phi$ preserves adjacencies, so these Cayley graphs are isomorphic as undirected graphs.

Thus, the planarity of Artin groups can be related directly to that of Coxeter groups. We have

Theorem 3. Given a graph $M$ with all edge labels either 2 or $\infty$, the Cayley graph of $\mathscr{A}(M)$ is planar if and only if each vertex of $M_{\infty}$ has degree 0 or 1 , and it has a planar embedding with no accumulation points of vertices if and only if $M_{\infty}$ consists of either isolated vertices (so the Artin group is free) or of two vertices joined by an edge (so the Artin group is $Z \times Z$ ).

\section{REFERENCES}

1. K. Appel and P. Schupp, Artin groups and infinite coxeter groups, Invent. Math. 72 (1983), 201-220.

2. J. Birman, Braids, links and mapping class groups, Princeton Univ. Press, Princeton, NJ, 1974.

3. E. Brieskorn and K. Saito, Artin-Gruppen and Coxeter-Gruppen, Invent. Math. 17 (1972), 245-271.

4. H. S. M. Coxeter and W. O. J. Moser, Generators and relations for discrete groups, 3rd ed., Springer, New York, 1972.

5. F. Harary, Graph theory, Addison-Wesley, Reading, MA, 1969.

Department of Mathematics, James Madison University, Harrisonburg, VIRGINIA 22807

E-mail address: FAC_DROMS@VAX1.ACS.JMU.EDU

Department of Mathematics, Massachusetts Institute of Technology, Cambridge, MASSACHUSETTS 02139

E-mail address: JHS@PASCAL.MIT.EDU 